\title{
Heart-Lung Transplant in Congenitally Corrected Transposition of the Great Arteries and Dextrocardia Patient
}

\author{
Magdy El-Sayed Ahmed ${ }^{1}$, Mathew Thomas ${ }^{1}$, Ian Makey ${ }^{1}$, Archer Martin ${ }^{1}$, David Erasmus \\ ${ }^{1}$, Basar Sareyyupoglu ${ }^{1}$, Kevin Landolfo ${ }^{1}$, and Si Pham ${ }^{1}$ \\ ${ }^{1}$ Mayo Clinic Florida
}

July 16, 2020

\begin{abstract}
Background and aim of the study: Congenitally corrected transposition of the great arteries (cc-TGA) is a rare type of congenital heart disease and heart-lung transplant (HLTx) is the potential solution if the patient developed severe biventricular failure.. Aim of the study is to present a challenging case of cc-TGA with dextrocardia managed by HLTx. Methods: A 53-year-old male patient was presented to our institution with biventricular failure. The echocardiogram revealed cc-TGA, dextrocardia with situs solitus, atrioventricular discordance and ventriculoatrial discordance, severe systemic and sub-pulmonary atrioventricular valves regurgitation, and severe pulmonary hypertension. He underwent heart-lung transplant. Results: The patient was discharged on postoperative day 25 on room air with normal left ventricular ejection fraction. Conclusion: This case highlights the complexity of preoperative and intraoperative management of cc-TGA, and demonstrates the challenges which the surgeons and anesthesiologists may face during the procedure of HLTx.
\end{abstract}

\section{Introduction}

Congenitally corrected transposition of the great arteries (cc-TGA) is a rare type of congenital heart disease with an incidence of less than $1 \%$ of infants with congenital heart disease ${ }^{1}$. Other terms have been used to describe this congenital anomaly such as L-TGA, double discordance and ventricular inversion. The first heart-lung transplantation (HLTx) was performed at Stanford University in $1981 .^{2}$ Pulmonary vascular disease and cystic fibrosis were the main indications for HLTx initially. However, in the recent years, the indication has been expanded to include congenital heart disease and idiopathic pulmonary artery hypertension. ${ }^{3}$ We report a rare case of cc-TGA with dextrocardia presenting with biventricular failure who underwent HLTx.

\section{Materials and Methods}

A 53-year-old male patient presented to our institution with the clinical picture of biventricular failure. The echocardiogram revealed cc-TGA, dextrocardia with situs solitus, atrioventricular discordance and ventriculoatrial discordance (figure $1 \mathrm{~A} \& \mathrm{~B}$ ), severe systemic (morphologic tricuspid valve) and sub-pulmonary atrioventricular (morphologic mitral valve) valves regurgitation, and severe pulmonary hypertension $(\mathrm{PH})$ (mean pulmonary artery pressure: $51 \mathrm{mmHg}$ ). His past medical history was remarkable for complete heart block mandating pacemaker implantation, and atrial fibrillation. The patient was evaluated and was deemed a candidate for HLTx. A 25-year-old male donor who died of hemorrhagic stroke became available and the en-bloc heart-lung was recovered by an experienced team (figure $2 \mathrm{~A}$ ).

The patient underwent HLTx via a clamshell incision. Because of hemodynamic instability, we commenced cardiopulmonary bypass (CPB) with an aortic cannula and a dual-stage venous cannula placed in the right atrium. After the heart was decompressed, the venous cannulation was converted from atrial into bi-caval configuration. 
Cardiectomy was performed, including complete removal of right and left atria, leaving a stump of atrial tissue on the inferior vena cava. The recipient trachea was resected one tracheal ring above the carina. The left recurrent laryngeal and the vagus nerves were identified and preserved during dissection. The two phrenic nerves were identified and protected by leaving pericardial flaps on each side. Tracheal anastomosis was performed first followed by the IVC, aorta and then the superior vena cava. The aortic cross clamp was removed and the patient was safely weaned from the CPB. Inotropic support was initiated using Dobutamine at $5 \mathrm{mcg} / \mathrm{kg} / \mathrm{min}$ and epinephrine at $0.05 \mathrm{mcg} / \mathrm{kg} / \mathrm{min}$. At the end of the procedure, the patient was taken to the intensive care unit in a stable condition.

The ischemic time was 296 minutes The CPB time was 280 minutes.

The standard immunosuppression protocol was initiated:

1- Methylprednisone $1 \mathrm{~g}$ IV intraoperatively, then $125 \mathrm{mg}$ bid for the first day, then $1 \mathrm{mg} / \mathrm{kg} /$ day in divided doses for 3 days then prednisone $20 \mathrm{mg}$ daily for 4 weeks then $10 \mathrm{mg}$ daily.

2- Mycophenolate 1g IV intraoperatively, followed by $500 \mathrm{mg}$ bid. Dose was temporarily increased to 1000 mg bid on POD 18 with suspicion of humoral rejection.

3- Single dose induction with Alemtuzumab $30 \mathrm{mg}$ IV administered on POD 1.

4- Tacrolimus $0.15 \mathrm{mg} / \mathrm{kg} /$ day dose titrated to achieve level of $6-10 \mathrm{mcg} / \mathrm{L}$.

On postoperative day 2 the patient was extubated and ambulation was initiated. The patient progressed steadily and ready for discharge, however on POD 18, he developed pleural effusion and lung infiltrates. Transbronchial lung biopsy revealed focal vasculitis. The diagnosis of possible humoral rejection was considered and the patient received a single dose of $500 \mathrm{mg}$, methylprednisone and 5 daily sessions of plasma exchange. Also he developed gastroparesis which completely resolved before discharge. Postoperative chest radiograph showed the heart and lungs in the proper position (figure $2 \mathrm{~B}$ ).

\section{Results}

The patient was discharged on postoperative day 25 on room air with left ventricular ejection fraction of $60-65 \%$.

\section{Discussion}

Over the past 20 years, the number of HLTx has been decreasing from 145 in 1999 to 45 in 2019 and gradually replaced with bilateral lung transplant (BLTx). ${ }^{4}$ It has been an ongoing debate on whether to perform HLTs or BLTx for patients with $\mathrm{PH} .{ }^{5} \mathrm{HLTx}$ is recommended to patients with congenital heart disease and Eisenminger syndrome, severe right ventricular (RV) dysfunction, and severe left ventricular dysfunction. On the other hand, BLTx provides comparable outcomes, moreover, it offers the advantage of better organ sharing. Given the long waiting time, patients with $\mathrm{PH}$ and simple cardiac anomalies such as atrial septal defect, patent ductus arteriosus or perimembranous ventricular septal defect can be successfully managed with a combination of BLTx and cardiac repair. The cut-off values for BLTx can be approximated at $10-25 \%$ RVEF and $32-55 \%$ LVEF. For values less than this cut-off, HLTx is recommended. ${ }^{5}$

We are reporting a successful HLTx in a patient with cc-TGA and dextrocardia. Our patient was presented with advanced cardiac problems including severe $\mathrm{PH}$, severe systemic and sub-pulmonary atrioventricular valves regurgitation, complete heart block and biventricular failure. In our patient, the RV was the systemic ventricle and its failure attributed to the severely incompetent systemic valve and the afterload systemic pressure. Hence, BLTx will not improve the condition of the failing systemic RV and the HLTx was deemed the suitable option. Based on the aforementioned findings, the multidisciplinary team recommended HLTx procedure. HLTx is technically a well-established surgical procedure, however, our case demonstrated unique surgical challenges due to the complexity of the congenital cardiac anomaly (cc-TGA and dextrocardia) of the recipient. The combination of the cc-TGA and dextrocardia is extremely rare. To the best of our knowledge, only one case of cc-TGA and dextrocardia has been reported by Takemoto et al. in the literature 
which was managed medically (i.e. no HLTx). ${ }^{6}$ This combination posed challenges for both the surgeon and anesthesiologist during the preoperative preparation and during the transplant procedure. The strategy of the approach, cannulation, and the technique was quite different from the standard HLTx. Giving the fact that both the superior and inferior vena cava were presented posteriorly, venous cannulation was initiated via dual stage venous cannula placed in the right atrium transitioned to bi-caval configuration after decompressing the heart.

According to the 2016 ISHLT report included the data of patients underwent HLTx in 2004-2014, the survival rates at $1,2,5$, and 10 years are $63 \%, 52 \%, 45 \%$, and $32 \%$, respectively, and a median survival of 5.8 years. $^{7}$ As of today, 15 months postoperatively, the patient is doing well.

\section{Conclusion}

This case highlights the complexity of preoperative and intraoperative management of cc-TGA, moreover, it demonstrates the challenges which the surgeons and anesthesiologists may face during the procedure of HLTx.

\section{Author Contributions}

Concept/design: MME, MT, SMP

Data analysis/interpretation: MME, MT, AKM, DBE, JCLM, KPL, SMP

Drafting article: MME

Critical revision of article: MME, MT, BS, AKM, IAM, DBE, KPL, SMP

Approval of article: MME, MT, AKM, IAM, BS, DBE, KPL, SMP

Statistics: N/A

Data collection: MME

\section{References}

1. Bjarke BB, Kidd BSL. Congenitally corrected transposition of the great arteries: a clinical study of 101 cases. Acta Paediatr Scand 1976;65:153-160.

2. Reitz BA, Wallwork JL, Hunt SA, et al. Heart-lung transplantation: successful therapy for patients with pulmonary vascular disease. N Engl J Med 1982;306:557-564.

3. Yusen RD, Edwards LB, Kucheryavaya AY, et al. The registry of the International Society for Heart and Lung Transplantation: thirty-first adult lung and heart-lung transplant report-2014; focus theme: retransplantation. J Heart Lung Transplant 2014;33:1009-1024.

4. Hill C, Maxwell B, Boulate D, et al. Heart-lung vs. double-lung transplantation for idiopathic pulmonary arterial hypertension. Clin Transplant 2015;29:1067-1075.

5. Olland A, Falcoz P, Canuet M, Massard G. Should we perform bilateral-lung or heart-lung transplantation for patients with pulmonary hypertension? Interact CardioVasc Thorac Surg 2013;17:166-170.

6. Takemoto M, Aoki K, Noma M, Yamamoto H. Congenitally corrected transposition of the great arteries with dextrocardia. Clin Cardiol. 2006; 29(1):44.

7. Yusen RD, Edwards LB, Dipchand AI, Goldfarb SB, Kucheryavaya AY, Levvey BJ, et al. The Registry of the International Society for Heartand Lung Transplantation: Thirty-third Adult Lungand HeartLung Transplant Report-2016; Focus Theme: Primary Diagnostic Indications for Transplant. J Heart Lung Tranplant. 2016;35:1170-1184.

\section{Figure Legends}

Figure 1: Preoperative chest radiograph showing the dextrocardia anomaly (A), and diagram showing anatomy of the congenitally corrected transposition of the great arteries with dextrocardia and situs solitus. RV: right ventricle, LV: left ventricle, RA: right atrium, LA: left atrium, IVC: inferior vena cava, SVC: superior vena cava (B) 
Figure 2 : Intraoperative photo showing the en bloc heart and lung after recovery (A), and postoperative chest radiograph showing the transplanted heart and lungs in the proper position (B).
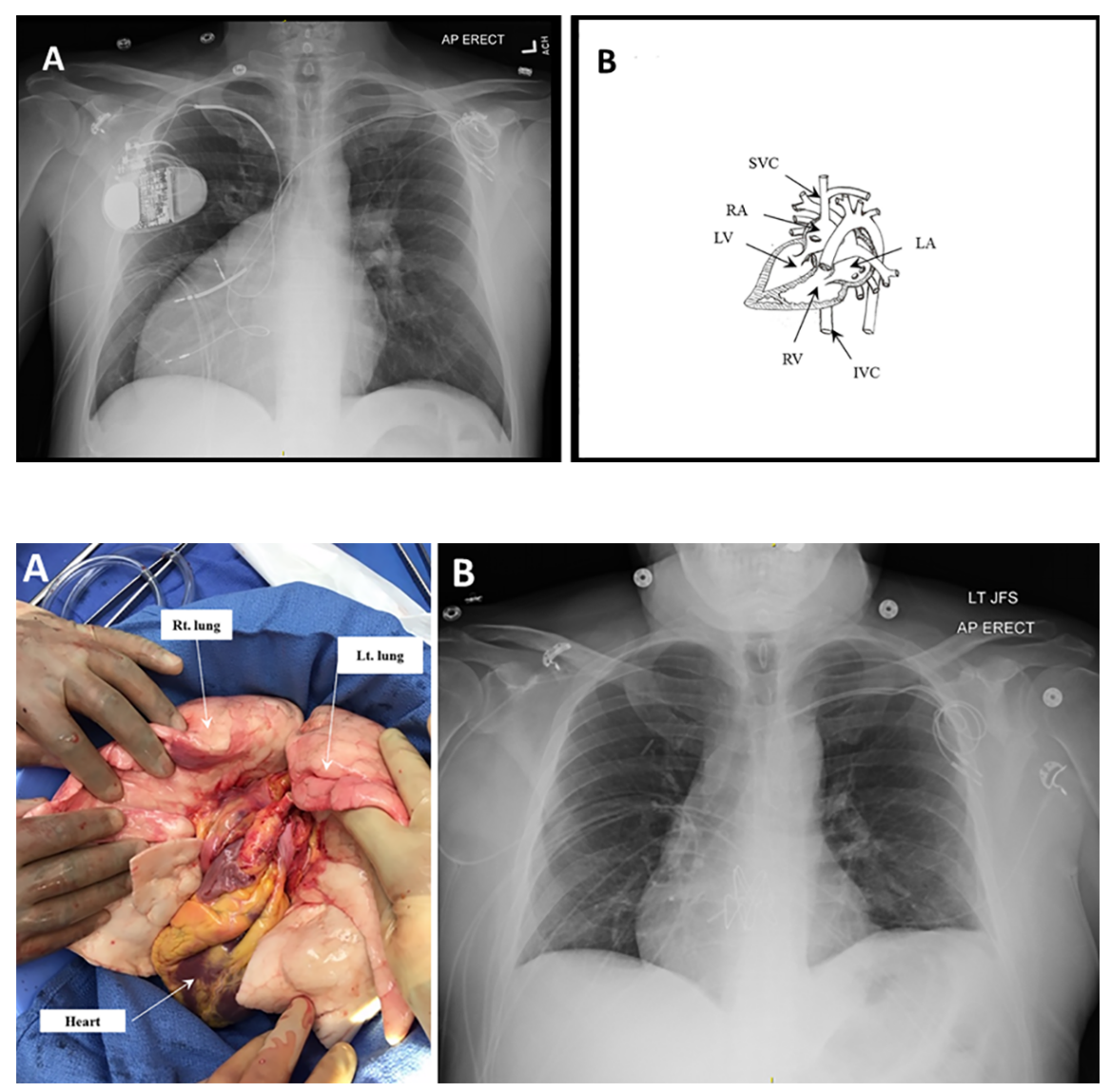